\title{
The "New Settlers": Results of a Bacteriological Survey During the First 6-Months Operation Period of an Internal Medicine Ward in a Tertiary Hospital
}

\author{
Gad Segal, Adi Brom and Erez Ramati \\ Internal Medicine “T”, Sheba Medical Center, Tel Hashomer, Israel
}

Received 2013-11-11; Revised 2013-11-14; Accepted 2013-11-27

\begin{abstract}
During the first six months of operating a new Internal-medicine ward in a tertiary hospital, a prospective survey, tracking both patient and environment bacteriological samples taken, was conducted. The motive behind the study was to establish the pattern in which environmental colonization is transmitted to the hospitalized patients and vice versa, thus defining the nosocomial pathogens that are typical to the ward. That information can be used to guide empirical antibiotic treatment. Patient sampling was done on clinical grounds whereas environmental cultures were systematically acquired from different surfaces around the ward. 6-months' results analysis suggest that clinically guided culture rates were tightly associated with volume of patients admitted, with no such association demonstrated between volume of cultures taken and rate of positive results, except for urine cultures. Regarding environmental sampling, we demonstrated no benefit in taking empirical, surface samples for common nosocomial pathogens. Our findings could further improve future resources allocation with regard to infection control and clinical bacteriology routines in newly established internal medicine wards.
\end{abstract}

Keywords: Bacteriological survey, Colonization, Cultures, Environmental Cultures, Internal Medicine

\section{INTRODUCTION}

As a part of an effort to document the unique process of establishing a new Internal Medicine ward in a tertiary hospital, we undertook a project of documenting and analyzing all bacteriologic cultures taken, both from patients and environment, during the first 6-months' period of our new, Internal Medicine ward in Tel Hashomer; Israel's largest tertiary medical center.

Since infectious diseases constitute a major part of our patients' milieu, we tracked and analyzed all cultures taken from bodily fluids, cultures that were taken as part of the routine management of patients suspected to have an infection: both blood and urine samples' taken and results' were documented. Also, we wanted to document the environmental pathogens "settling" within the department. Such documentation could potentially depict the pattern of such "settlements" and could be associated with clinical patterns of nosocomial infections' spreading amongst our patients.

Blood and urine cultures have a crucial role in the treatment of patients suspected to have infectious diseases, allowing for specific identification of the pathogen responsible and tailoring the required antibiotic regimen. Nevertheless, notwithstanding the fact that there is a wealth of information regarding the usefulness of specific cultures in specific clinical entities (e.g., pneumonia or urinary tract infections) less is known with regard to the rate at which cultures are acquired in a given, newly established Internal Medicine ward and the yield of positive cultures from total.

This study summarizes the total number of cultures, blood and urine, taken during routine clinical work in our ward during its first 6-months of operation, the Corresponding Author: Erez Ramati, Internal Medicine “T”, Sheba Medical Center, Tel Hashomer, Israel 
number of positive results received and the results of environmental cultures taken from various sites around the ward during that same period.We plotted the volume of different cultures taken and the rate of positive cultures against a time line, assuming and evaluating the correlation with our build-up increase of patients' numbers. We hope that our unique experience would benefit future, similar efforts.

\section{MATERIALS AND METHODS}

\subsection{Data Acquisition}

During the first six months of an internal medicine ward operation (dating from 20/1/2013 to 31/7/2013), all blood and urine cultures taken along with their results were routinely recorded. In parallel, we collected environmental cultures, to be assessed regularly at the same institutional laboratory.

\subsection{Culture Acquisition}

Blood samples were obtained by physiciansapplying standard aseptic techniques, using commercial blood culture kits (Becton Dickinson, Bactec Culture Vials, Ireland). Samples were regularly sent to the bacteriological lab for processing. The most common clinical association for blood sampling was febrile illness. Urine samples were collected by the nursing staff via mid-stream urine collection from or indwelling catheters, using commercial urine culture kits (Agit Technologies, Israel). Samples were regularly sent to the bacteriological lab for processing. The most common clinical association for urine sampling was febrile illness followed by urinary complaints and indwelling catheters. All samples taken from patients with indwelling catheters were attained only after catheter replacement according to accepted guidelines.Environmental samples were collected in intervals of 2 to 3 weeks each by a sole investigator, using swabs (COPAN Italia, Italy). Samples were attained in accord with accepted guidelines (opened in septic fashion, saline irrigated, brushed across surface 2-3 times) from predetermined surfaces in the ward's environment:

- Medical staff office-doorknobs, keyboards, light switches

- Nursing staff office-doorknobs, keyboards, light switches

- Men's wardrobe-doorknobs, light switches

- Admission counter

\subsection{Statistical Analysis}

Data analysis was made in order to establish or defy correlation of positive clinical cultures (blood and urine) with the mere volume of patients admitted on one hand and with the volume of cultures taken, on clinical grounds, on the other hand. Pearson correlation, with its potential statistical significance was analyzed using the SPSS software.

\section{RESULTS}

During a period of six months, subdivided into 19 checkpoints of 10 days each, a total of 928 patients were admitted to the ward. A total of 1,333 different biological sample-cultures were collected from patients, on clinical grounds, according to the physicians' deliberation, without any relation to our study (it could be stated that physicians were blinded to the study). A total of 285 clinical cultures $(21 \%)$ were positive for pathogenic bacteria. During the same period 12 environmental culture sets were also collected. Table 1 describes the rate of blood and urine samples taken alongside the rate of hospitalizations on the wards' time-line of increased admission volume.

\subsection{Blood Cultures}

As evident in Fig. 1, data analysis indicates that while a statistically significant correlation exists between the number of patients hospitalized and the number of cultures obtained (Correlation 0.82, $\mathrm{p}<0.001$ ), no such correlation exists between the number of cultures obtained and the number of positive results (Correlation 0.22, $\mathrm{p}=0.929$ ).

\subsection{Urine Cultures}

As evident in Fig. 2, data analysis indicates that a statistically significant correlation exists between the number of patients hospitalized and the number of cultures obtained (Correlation 0.52, $\mathrm{p}=0.021$ ). Unlike the results regarding blood cultures, there also exists a statistically significant correlation between the number of cultures obtained and the number of positive results (Correlation 0.89, $\mathrm{p}<=0.001$ ).

\subsection{Environment Cultures}

As evident in Fig. 3, data analysis indicates that while no statistically significant correlation exists between the number of patients hospitalized and the number of cultures obtained (Correlation-0.14, $\mathrm{p}=0.55$ ), there is such a correlation between the number of cultures obtained and the number of positive results (Correlation 0.48, $\mathrm{p}=0.036$ ). 
Gad Segal et al. / American Journal of Infectious Diseases 9 (4): 136-141, 2013

Table 1. Clinical and environment samples taken alongside the rate of total hospitalizations

\begin{tabular}{|c|c|c|c|c|c|c|c|}
\hline \multirow[b]{2}{*}{ Period } & \multicolumn{2}{|l|}{ Blood } & \multicolumn{2}{|l|}{ Urine } & \multicolumn{2}{|c|}{ Environment } & \multirow[b]{2}{*}{ Total hospitalized patients } \\
\hline & Obtained & Positive (\%) & Obtained & Positive (\%) & Obtained & Positive (\%) & \\
\hline 1 & 30 & 3 & 12 & $6(50)$ & 2 & 0 & 28 \\
\hline 2 & 23 & 2 & 6 & 3 & 1 & 0 & 23 \\
\hline 3 & 29 & 2 & 4 & 2 & 1 & 0 & 50 \\
\hline 4 & 24 & 0 & 10 & 9 & 0 & 0 & 28 \\
\hline 5 & 24 & 6 & 7 & 5 & 2 & 1 & 33 \\
\hline 6 & 31 & 9 & 11 & 3 & 0 & 0 & 47 \\
\hline 7 & 29 & 8 & 8 & 16 & 1 & 0 & 39 \\
\hline 8 & 35 & 3 & 15 & 15 & 1 & 0 & 48 \\
\hline 9 & 27 & 5 & 17 & 5 & 1 & 0 & 39 \\
\hline 10 & 33 & 2 & 10 & 16 & 1 & 0 & 38 \\
\hline 11 & 42 & 4 & 21 & 18 & 0 & 0 & 53 \\
\hline 12 & 42 & 2 & 28 & 18 & 1 & 0 & 51 \\
\hline 13 & 49 & 4 & 20 & 11 & 1 & 0 & 80 \\
\hline 14 & 41 & 4 & 19 & 9 & 0 & 0 & 72 \\
\hline 16 & 40 & 1 & 10 & 13 & 0 & 0 & 61 \\
\hline 17 & 42 & 4 & 20 & 6 & 0 & 0 & 58 \\
\hline 18 & 34 & 5 & 11 & 10 & 0 & 0 & 67 \\
\hline 19 & 42 & 3 & 16 & 14 & 0 & 0 & 47 \\
\hline 20 & 51 & 7 & 22 & 14 & 0 & 0 & 66 \\
\hline Total & 668 & $75(11.2)$ & 267 & $188(70.41)$ & 12 & $1(4.4)$ & 928 \\
\hline
\end{tabular}

—Total Positive Blood Cultures —Total Cultures — Hospitalized

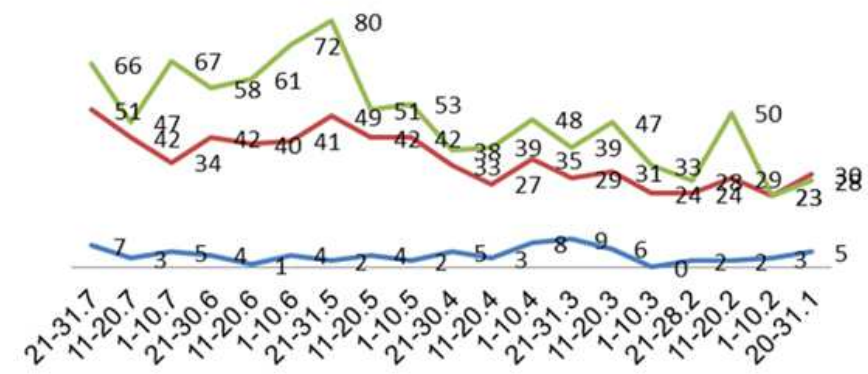

Fig. 1. Blood cultures taken plotted against positive results and total number of hospitalized patients

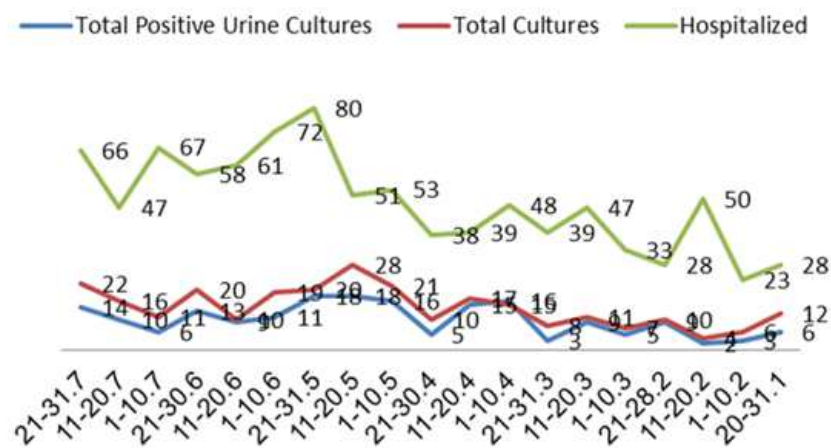

Fig. 2. Urine cultures taken plotted against positive results and total number of hospitalized patients 


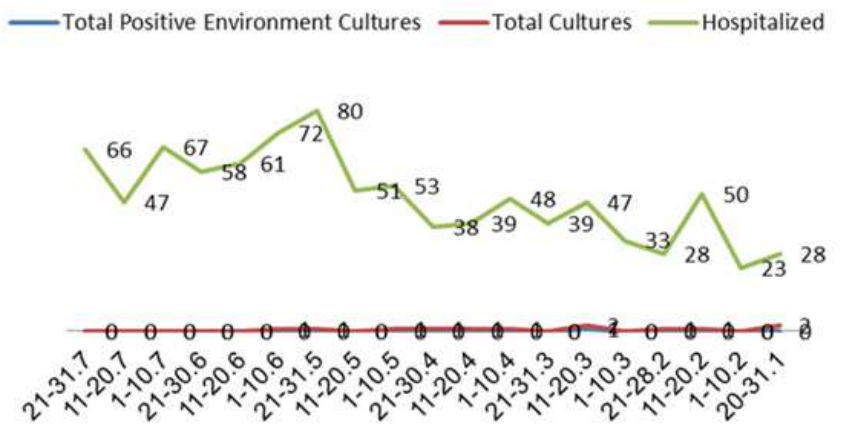

Fig. 3. Environment cultures taken plotted against positive results and total number of hospitalized patients

\section{DISCUSSION}

Infection is still the most common cause of death amongst patients admitted to internal medicine wards. In cases infection is suspected, cultures are obtained in order to identify the causative agent and to direct a specific and adequate antibiotic treatment. The literature demonstrates that despite its potential use, at least blood cultures are not sensitive enough.

A study conducted by Sato and his colleagues among internal medicine departments in Japan (Sato et al., 2012) shows a total isolation rate of only $11.8 \%$ from blood cultures taken. A similar study in Korea (Shin et al., 2011) reveals that the incidence of positive blood cultures was $10.1 \%$, with $2.1 \%$ attributable to contamination by skin flora and the rest to true pathogens. Similar findings were described in the pediatric population (Waltzman and Harper, 2001; McIntyre et al., 1987). Our study found similar results: a rate of $11.2 \%$ positive blood cultures, with no correlation between the number of cultures obtained and the number of positive cultures. Considering the correlation that was found between the number of the admitted patients and the number of cultures taken, it seems that the staff members obtain cultures based on high clinical suspicion rather than objective criteria.

Several reasons that may explain a false negative result in a blood culture, including prior antibiotic treatment that the patient receives when the sample is taken, the time interval between sampling, skin disinfection methods used before the culture is drawn, volume of the sample drawn and laboratory methods. The need to improve the sensitivity of the test while avoiding false positive results is discussed in the literature in different contexts in the non-surgical patients-emergency departments (Shapiro et al., 2008), hospitalization wards (Coburn et al., 2012; David et al., 1990) and ICU (Shafazand and Weinacker, 2002).
In a review by Coburn and co. regarding febrile immune-competent patients in whom blood cultures were taken, very low rate of true bacteremia (4-7\%) were recorded. Therefore, their recommendation is to carefully weigh the expected benefits of taking blood cultures versus the clinical presentation of the patient. In order to improve the expected yield, they proposed 3 bacteremia risk groups-low, medium and high-depending on the estimated diagnosis upon admission, including statistically proven clinical markers which can help decide whether or not blood culture should be taken, or for interpreting the result of an empirically obtained positive blood culture. Such criteria are currently not applied in our institution.

Urine cultures are frequently obtained as part of the investigation for possible infection in the hospitalized patient. The rates of positive urine cultures vary between 9-12\% (Kader et al., 2004; Kenneth and Hyams, 1987). It's important to note that Bacteruria is highly affected by the antibiotic treatment the patient receives; after a treatment course is given, the rate of positive cultures greatly declines, as well as the rationale of obtaining the sample in the first place (Kenneth and Hyams, 1987). Various studies show that it's not always possible to establish a connection between Bacteruria and symptomatic urinary tract infection.Urine cultures are sometimes taken in patients presenting with fever or confusion suspected for infectious origin. However, positive results do not necessarily indicate a urinary tract infection or an infection at all (Cope et al., 2009). Therefore, Bacteruria in the hospitalized patient is not an indication to begin antibiotic therapy, in patients with or without a catheter (Silver et al., 2009). Bacterial load in the urine culture can be used in an attempt to prove a link between the patient's clinical presentation and Bacteruria; One study showed that appearance of more 
than 100,000 colonies per culture increased the incidence of symptomatic urinary tract infection by a factor of 73 (Kwon et al., 2012). This study's authors believe that setting the threshold of positive culture at 100,000 colonies will decrease the incidence of positive urine cultures by $38 \%$ and thereby reduce unnecessary and costly antibiotic treatments.Our study demonstrated a correlation between the number of hospitalized patients to the number of urine culture that were taken, as well as a correlation between them and the number of positive cultures. Overall $70.1 \%$ were positive-a much higher rate than that described in the literature. The high incidence can be related to high contamination rate, low threshold for defining "positive culture", or a too strict policy regarding the decision to obtain urine cultures in our ward.

Environmental cultures are described in the literature as an acceptable method to characterize the population (Ake et al., 2011; Rastogi et al., 2012) and the resistance profile (Huang et al., 2012) of bacteria among recently opened wards and also in establishing a link between environmental pathogens and patients' infections in a temporary medical ward under field conditions (Scott et al., 2007). In this study we investigated whether there is a relationship between the bacteria population that was isolated from the various clinical cultures over time and the results of environmental cultures. Of all the environmental cultures taken, we received a single growth of MSSA, attributed to contamination of the culture. No pathogen associated with life threatening nosocomial infection was isolated. A possible explanation is the examined specimen surfaces selected. The literature describes evidence for presence of pathogenic flora on bed linen (Sui et al., 2012) and water sources (Yaslianifard et al., 2012). It may also be beneficial to sample staff members and their personal equipment (Marinella et al., 1997; Stewart et al., 2011), none of these was examined in the current study. As before, another possible explanation may lie in sampling techniques and laboratory methods.

The study has several limitations. No standard criteria were set determine when it is or isn't appropriate to take cultures and the decision was made according to the clinical judgment discretion of the department's physicians, based on the patient's presentation and objective measures such as fever and increased white blood cell counts. It may have led to taking unnecessary cultures, resulting in a decreased rate of overall positive cultures. Additionally, technical problems can probably affect the final results-for example lack of uniformity in aseptic technique or failure of sample volume. Some limitation are unique to culture type; as for blood cultures, there is no reference to sub-populations, since according to the literature some diseases and conditions tend to result in higher rates of bacteremia and accordingly higher percentage of positive blood cultures. Urine cultures were sometimes taken from patients with an indwelling permanent catheter, or after several days with a temporary catheter (inserted during hospitalization), therefore growth of bacteria rather reflects the flora accumulated on the catheter and does not necessarily indicate an active infection.

Regarding all types of clinical cultures, many patients admitted to the department after an antibiotic course received in the community, sometimes even a prolonged one and most of patients with suspected infectious diseases receive an antibiotic treatment in the emergency department prior to hospitalization. This undoubtedly reduces to growth rate in the cultures.

\section{CONCLUSION}

This study demonstrates that environmental specimens obtained from various surfaces using the methods described correlate poorly with the flora obtained from patient cultures during the same time period. Also, our results suggest that the rate at which bacteriological specimens are acquired is mainly a function of patient volume within the ward. While blood cultures generally show low growth rate, urine cultures had a surprisingly high rate. Both of these findings probably contain inaccuracies resulting from acquisition technique and prior antibiotic therapy. It is possible that adoption of standard protocols aimed at careful patient selection and culture technique could improve the validity of blood and urine cultures and warrants additional research.

\section{REFERENCES}

Ake, J.M.D., P. Scott, M.D. MPH, G. Wortmann and M.D. Xiao-Zhe et al., 2011. Gram-negative multidrug-resistant organism colonization in a US military healthcare facility in Iraq. Infect. Control Hosp. Epidemiol., 6: 545-545. DOI: 10.1086/660015

Coburn, B., A.M. Morris, G. Tomlinson and A.S. Detsky, 2012. Does this adult patient with suspected bacteremia require blood cultures. JAMA, 308: 502511. DOI: $10.1001 /$ jama.2012.8262 
Cope, M., M.E. Cevallos, R.M. Cadle, R.O. Darouiche and Musher et al., 2009. Inappropriate treatment of catheter-associated asymptomatic bacteriuria in a tertiary care hospital. Clin. Infect. Dis., 1: 11821188. DOI: $10.1086 / 597403$

David, W., M.D. Bates, E.F. Cook, L. Goldman and M,D. MPH et al., 1990. Predicting bacteremia in hospitalized patients. A prospectively validated model. Ann. Intern. Med., 1: 495-500. DOI: 10.7326/0003-4819-113-7-495

Huang, X.Z., J.G. Frye, M.A. Chahine, L.M. Glenn and J.A. Ake et al., 2012. Characteristics of plasmids in multi-drug-resistant Enterobacteriaceae isolated during prospective surveillance of a newly opened hospital in Iraq. PLoS One, 7: e40360-e40360. PMID: 22808141

Kader, A.A., A. Kumar and S.M. Dass, 2004. Antimicrobial resistance patterns of gram-negative bacteria isolated from urine cultures at a general hospital. Saudi J. Kidney Dis Transpl., 15: 135-9. PMID: 17642764

Kenneth, C. and M.D. Hyams, 1987. Inappropriate urine cultures in hospitalized patients receiving antibiotic therapy. Arch. Intern. Med., 147: 48-49. DOI: 10.1001/archinte.1987.00370010052013

Kwon, J.H., M.K. Fausone, H. Du, A. Robicsek and L.R. Peterson, 2012. Impact of laboratory-reported urine culture colony counts on the diagnosis and treatment of urinary tract infection for hospitalized patients. Am. J. ClinPathol., 137: 778-84. DOI: 10.1309/AJCP4KVGQZEG1YDM

Marinella, M.A., M.D. Carl Pierson and C. Chenoweth, 1997. The stethoscope. A potential source of nosocomial infection? Arch. Intern. Med., 157: 78690. DOI: 10.1001/archinte.1997.00440280114010

McIntyre, P.B., M.H. Tilse, M. O'Callaghan and J.G. McCormack, 1987. Blood cultures in hospitalized children. Med. J. Aust.,147: 485-9. PMid:3500392

Rastogi, S., M.D. MMMemail, A.R. Shah, M.D. Jason Perlman and M.D.A. Bhutada et al., 2012. Pattern of bacterial colonization in a new neonatal intensive care unit and its association with infections in infants. Am. J. Infect. Control, 40: 512-515. DOI: 10.1016/j.ajic.2012.02.016

Sato, R., H. Miyamoto, Y. Aoki, S. Hayashi and T. Mizuta et al., 2012. Characteristics of bacterial species in positive blood cultures among hospitalized patients in three wards in the Department of Internal Medicine: retrospective chart review 1999-2008. Int. Med., 51: 1159-1166. PMID: 22687784
Scott, P., G. Deye, A. Srinivasan, C. Murray and K. Moran et al., 2007. An outbreak of multidrugresistant Acinetobacter baumannii-calcoaceticus complex infection in the US military health care system associated with military operations in Iraq. Clin Infect Dis., 44: 1577-1584. DOI: $10.1086 / 518170$

Shafazand, S. and A.B. Weinacker, 2002. Blood cultures in the critical care unit: Improving utilization and yield. Chest, 122: 1727-1736. PMID: 12426278

Shapiro, N.I., R.E. Wolfe, S.B. Wright, R. Moore and D.W. Bates, 2008. Who needs a blood culture? A prospectively derived and validated prediction rule. J. Emerg. Med., 35: 255-64. PMID: 18486413

Shin, J.H., S.A. Song, M.N. Kim, N.Y. Lee and E.C. Kim et al., 2011. Comprehensive analysis of blood culture performed at nine university hospitals in Korea. Korean J. Lab. Med., 31: 101-106. DOI: 10.3343/kjlm.2011.31.2.101

Silver, S.A., L. Baillie and A.E. Simor, 2009. Positive urine cultures: A major cause of inappropriate antimicrobial use in hospitals. Can. J. Infect. Dis. Med. Microbiol., 20: 107-111. PMID: 21119801

Stewart, S., O. Blatchford and J. Reilly, 2011. Should healthcare workers be screened routinely for meticillin-resistant Staphylococcus aureus? A review of the evidence. J. Hosp. Infect., 4: 285-289. DOI: $10.1016 /$ j.jhin.2010.09.038

Sui, W., J. Wang, H. Wang, M. Wang and Y. Huang et al., 2012. Comparing the transmission potential of Methicillin-resistant Staphylococcus aureus and multidrug-resistant Acinetobacter baumannii among inpatients using target environmental monitoring. Am. J. Infect. Control, 41: 411-415. DOI: 10.1016/j.ajic.2012.08.007

Waltzman, M.L. and M. Harper, 2001. Financial and clinical impact of false-positive blood culture results. Clin. Infect. Dis., 33: 296-299. DOI: $10.1086 / 321881$

Yaslianifard, S., A.M. Mobarez, B. Fatolahzadeh and M.M. Feizabadi, 2012. Colonization of hospital water systems by Legionella pneumophila, Pseudomonas aeroginosa and Acinetobacter in ICU wards of Tehran hospitals. Ind. J. Pathol. Microbiol., 55: 352-356. DOI: 10.4103/0377-4929.101743 